\title{
Prospects of plasmapheresis for patients with severe COVID-19
}

\author{
Ilmira R. Gilmutdinova, Maksim Yu. Yakovlev, Petr S. Eremin, Anatoliy D. Fesun
}

Federal State Budgetary Institution "National Medical Research Centre for Rehabilitation and Balneology" of the Ministry of Health of Russia, Moscow, Russia

This article is distributed under the terms of the Creative Commons Attribution Noncommercial License (CC BY-NC 4.0) which permits any noncommercial use, distribution, and reproduction in any medium, provided the original author(s) and source are credited.

\begin{abstract}
On February 11, 2020, the World Health Organization officially named the infection caused by the new coronavirus "Coronavirus disease 2019" (COVID-19). On February 11, 2020, the International Committee on Taxonomy of Viruses (ICTV) officially named the infectious matter "severe acute respiratory syndrome coronavirus 2" (SARS-CoV-2). Emergence of severe complications with new coronavirus disease is due to the development of hypercytokinaemia, also known as "cytokine storm". These complications comprise acute respiratory distress syndrome (ARDS), respiratory failure and death. Emerging data point to the logic of using extracorporeal haemocorrection to normalise cytokine levels and reduce the severity of organ disorders. The analysis of the literature indicates that to date, a certain positive experience has been accumulated in the world in the application of extracorporeal methods in clinical practice in patients with COVID-19.
\end{abstract}

Key Words: plasmapheresis, extracorporeal haemocorrection, COVID-19, coronavirus.

On February 11, 2020, the World Health Organization officially named the infection caused by a new coronavirus "Coronavirus disease 2019" (COVID-19) and the International Committee on Taxonomy of Viruses (ICTV) officially named the infectious matter "severe acute respiratory syndrome coronavirus 2" (SARS-CoV-2). Throughout the ongoing COVID-19 pandemic, over $13.8 \%$ of cases are severe and over $6.1 \%$ are critical. ${ }^{1}$ The severity of the condition in patients of these subgroups is caused, interalia, by the development of hypercytokinaemia, also known as "cytokine storm". 2,3

\section{Hypercytokinaemia}

Cytokine Storm Syndrome (hypercytokinaemia) is a severe uncontrolled immune response, when cells of the immune system release excessive amounts of cytokines (interferons, interleukins, chemokines, colony stimulating factors, tumour necrosis factors, etc.) into the blood. ${ }^{2,4}$ For instance, a study published in Lancet Journal by Chinese scientists showed an increase in inflammatory cytokines/chemokines (IL-2, IL-7, IL-10, GCSF, IP-10, MCP-1, MIP1A and TNF- $\alpha$ ) in patients with severe and critical COVID-19 infection. ${ }^{5,6}$ It has been proven that the severity of COVID-19 disease is directly related to the cytokine storm and the amount of pro-inflammatory agents. ${ }^{7}$ Under normal conditions, levels of inflammatory and anti-inflammatory cytokines in the organism are balanced. However, when the virus implants into the body, the general immune response is triggered by activation of neutrophils, dendritic cells, macrophages, lymphocytes and natural killer cells, along with initiation of the local immune response. Activated cells release a large number of cytokines, especially of inflammatory type, triggering immune cells, which, in turn, release new cytokines. This positive feedback effect causes hyperexpression of cytokines in response to the etiological stimulus and the development of "cytokine storm". 2,4 This phenomenon is most often observed in patients with chronic diseases, compromised immunity and deep hypoxia. Such patients are more likely to develop acute respiratory distress syndrome (ARDS) and multiple organ dysfunction syndrome (MODS). In addition, they demonstrate high levels of C-reactive protein (CRP) and IL-6, a decrease in the number of lymphocytes, as well as abnormal hemostasis indicators. ${ }^{8}$ Thus, the world medical community is challenged to select therapeutic methods that allow to decrease the amount of inflammatory cytokines/chemokines and, consequently, to abate the cytokine storm, which aggravates the course of COVID-19 disease. ${ }^{6}$ Emerging data point to the logic of using extracorporeal haemocorrection to normalise cytokine levels and reduce the severity of organ disorders primarily in severe and critical ill patients. ${ }^{1,6,8-10}$ However, the use of extracorporeal haemocorrection methods in less severe 
patients is also possible. So, in scientific publications there are already positive results of the use of simple plasmapheresis, plasma exchange with of convalescent plasma and plasmapheresis with immunoglobulin therapy. ${ }^{11}$

\section{Plasmapheresis}

Plasmapheresis is the procedure of removal, treatment, and return of blood plasma or components thereof from and to the blood circulation. Plasmapheresis can be either therapeutic (extracorporeal methods of blood treatment by removing some plasma components) or donated (donated plasma is obtained from stored whole donor blood with subsequent return of cell blood elements). ${ }^{12-14}$ Plasmapheresis has been used for treatment of various diseases for many years. Apheresis Applications Committee of the American Society for Apheresis (ASFA) identified 4 groups of diseases, for treatment of which plasmapheresis can be used. The $1^{\text {st }}$ group includes diseases that reveal maximum efficiency of treatment with plasmapheresis. The $4^{\text {th }}$ group is for diseases, plasmapheresis treatment of which is inefficient. For instance, plasmapheresis proved best as part of a comprehensive treatment program for kidney diseases (glomerulonephritis, Goodpasture syndrome, focal segmental glomerulosclerosis, kidney transplantation etc.), in liver transplantation (desensitization), for treatment of myasthenia, thrombotic microangiopathy, thrombocytopenic purpura, Wilson disease, antiphospholipid syndrome, drug overdose, intoxications, systemic lupus erythematosus and various other diseases. ${ }^{15-19}$

In accordance with the classification of methods of extracorporeal haemocorrection (Sokolov AA, 2017) ${ }^{20}$ the following methods relate to the effect on blood plasma:

- membrane technology (plasma filtration, selective plasma filtration or selective plasma exchange, cascade plasma filtration, plasma diafiltration)

- centrifuge technology (plasmapheresis)

- $\quad$ sorption technology (plasmosorption) and others. ${ }^{20}$

In terms of efficiency and safety in regard to severe and critical patients, the procedure of selective and cascade plasma filtration has proved to be the most effective and safe of all. This technique is a method of extracorporeal haemocorrection based on the principle of filtration and convection plasma mass transfer through a semipermeable membrane due to the pressure gradient, which contributes to the effective removal of highmolecular large-globular blood plasma components exceeding the size of the albumin molecule. The plasma itself is obtained from whole blood through centrifugal or membrane plasmapheresis. ${ }^{17,20,21}$

Ig M, circulating immune complexes, cryoglobulins, some viruses and other large molecules and supramolecular complexes are effectively removed. ${ }^{13,20,22}$ Selective plasma filtration may be accompanied by a plasma exchange procedure in which the exfused volume is replaced by donor plasma. In this case, donor convalescent plasma containing antibodies against SARS-CoV-2 is used. ${ }^{23-25}$ The use of anti-COV2 immunoglobulins, which are contained in the COVID19 convalescent plasma, is a highly logical therapeutic approach aimed at forming a passive immunity. It should be noted that the safety and effectiveness of this COVID19 treatment method has not yet been fully confirmed. An international survey was done to question centers that studied the use of convalescent plasma in treatment of COVID-19 patients. The survey identified 64 research studies in 22 countries. However, only 20 centers from 12 countries revealed information on their studies. Out of all studies, only 4 are set to comprise 400 or more patients. The authors of this research express a hope that blind studies, randomization and larger sampling would allow these studies to provide objective results of high level of evidence. ${ }^{26}$ Clinical studies on this matter are being conducted in China, USA, Russia, France, and some other countries. However, some scientists have already published intermediate data indicating the positive effects of convalescent plasma in the treatment of COVID-19.23,27 The procedure of donor plasmapheresis is not only safe for donors themselves, but also useful in terms of improving blood rheology and correcting hypercoagulation. This effect is achieved through the use of anticoagulants based on citrate and removal of high-molecular blood components that increase blood viscosity. ${ }^{28}$ It is widely known that the majority of patients with COVID-19 have a hypercoagulation condition characterised by an increase in prothrombin time, D-dimer and fibrinogen levels at practically normal values of activated partial thromboplastin time. ${ }^{29,30}$

\section{Operating principles of plasmapheresis methods}

Figure 1 shows the basic operating principles of plasmapheresis methods in patients with severe form of COVID-19, that mainly include the following: ${ }^{8,31,32}$

- removing pathogenic circulating molecules, inflammatory cytokines;

- removing metabolic products such as creatinine, urea nitrogen;

- maintaining hemodynamic stability in the organism;

- correction of blood rheological disorders;

- correction of electrolyte and acid-alkaline balance disorders to maintain stability of internal environment

\section{Case reports and reccommendations}

Chinese scientists presented the results of treatment of three patients in severe condition with bilateral pneumonia and confirmed COVID-19 in Wuhan, China in the paper published in early April 2020. ${ }^{1}$ ] One of the patients underwent 3 plasma exchange sessions and two patients underwent plasma filtration using oXiris ${ }^{\circledR}$ hemofilter (Baxter, Meyzieu, France) modified with 
The basic operating principles of plasmapheresis methods in patients with severe form of COVID-19 mainly include the following: ${ }^{8,31,32}$

- removing pathogenic circulating molecules, inflammatory cytokines;

- removing metabolic products such as creatinine, urea nitrogen;

- maintaining hemodynamic stability in the organism;

- correction of blood rheological disorders;

- correction of electrolyte and acid-alkaline balance disorders to maintain stability of internal environment

Fig 1. Operating principles of plasmapheresis methods

AN69 adsorbing membrane. Two out of three patients recovered. One patient died due to complications (DICsyndrome and right-sided pneumothorax) and delayed application of plasmafiltration. This study reveals the importance of timely use of plasmapheresis and conveys the suggestion to do a separate research on efficiency of plasmapheresis in treatment of COVID-19 (considering the research timing). A retrospective study of the effectiveness and safety of plasma exchange in septic shock with multiple organ dysfunction was conducted at the Intensive Care Unit of Lexington Medical Centre (South Carolina, USA), involving 80 patients. The mortality rate in pneumonia patients treated with plasma exchange as an additional method of treatment amounted to $47.8 \%$ against $81.3 \%$ in the standard therapy group $(p<0.05) .{ }^{33}$ In the article published following the results of the study, the scientists note that they revised approaches to the use of plasma exchange method on the basis of the experience gained. Thus, they now recommend this method not only as a "rescue therapy", but also as part of earlier stages of treatment. ${ }^{9}$ In a published article professor Shi and his colleagues provided results on treatment of 50-year-old critically ill patient with COVID-19. Due to inefficient drug therapy, this patient received a course of plasma exchange followed by intravenous injections of immunoglobulin. The chosen treatment method allowed to stop the disease progression and lower the need for intensive supportive therapy. ${ }^{34}$ This case requires a larger clinical study including the use of immunoglobulin, apart from plasmapheresis. On April 10, 2020, the U.S. Food and Drug Administration (FDA USA) issued an emergency use authorisation for Terumo BCT Inc. and Marker Therapeutics AG to use their Spectra Optia Aphereis blood purification systems and Depuro D2000 adsorption cartridges to treat laboratory-confirmed COVID-19 patients over 18 years of age admitted to the ICU with respiratory failure syndrome. The purpose of use is the reduction of inflammatory cytokines concentration. According to the manufacturers' statement, the
Indications for an urgent start of treatment:

- clinical signs of progressive respiratory failure;

- signs of severe coronavirus infection (respiration rate $\geq 30 / \mathrm{min}$ and/or blood oxygen saturation $\geq 93 \%$ and/or $\mathrm{PO} 2 / \mathrm{FiO} 2$ index $\geq$ $200 \mathrm{~mm} \mathrm{Hg}$ );

- progression of lung damage at the rate of $\geq 10 \%$ per day, identified using one of the imaging methods;

- progressive increase of inflammatory marker levels

Fig 2. The choice of methods depends primarily on the capabilities of the medical staff

adsorption cartridge D2000 is effective in reducing the level of IL-3, IFN-gamma, IL-10, IL-1B, IL-6, IL-8, MCP-1 and TNF-alpha. ${ }^{35}$ The use of FDA-approved technology is allowed for patients over 18 years of age who have any of the following symptoms: i) early acute lung injury/early acute respiratory distress syndrome; or ii) severe health condition characterized by: ${ }^{35}$ respiratory embarrassment; respiratory rate $\geq 30 / \mathrm{min}$; $\mathrm{SpO}_{2}$ level $\leq 93 \%$; oxygenation (respiratory) index $<300$, and/or increase in lung infiltrates $>50 \%$ within $24-48$ hours; iii) critical health condition, determined by: respiratory failure; toxic shock syndrome and/or multiple organ dysfunction.

The issue of using plasmapheresis technologies was also addressed in the Russian Federation. Thus, the Scientific Society of Experts of Extracorporeal Blood Treatment in Intensive Carepresented interim recommendations "on the use of methods of extracorporeal haemocorrection in the complex treatment of patients with new coronavirus disease COVID-19". According to these recommendations, a key point of reaching the maximum efficiency of the extracorporeal haemocorrection technique is its early application, namely, during the early stages of clinical and laboratory manifestation of cytokine storm syndrome and multiple organ dysfunction.

Below are indications for an urgent start of extracorporeal treatment (Figure 2):

- clinical signs of progressive respiratory failure;

- $\quad$ signs of severe coronavirus infection (respiration rate $\geq 30 / \mathrm{min}$ and $/$ or blood oxygen saturation $\leq$ $93 \%$ and/or $\mathrm{PO}_{2} / \mathrm{FiO}_{2}$ index $\leq 200 \mathrm{~mm} \mathrm{Hg}$ );

- progression of lung damage at the rate of $\geq 10 \%$ per day, identified using one of the imaging methods;

- $\quad$ progressive increase in inflammatory marker levels (CRB, IL-6 etc.)

Following these recommendations, the choice of extracorporeal haemocorrection method depends 
primarily on the capabilities of the medical staff as well as on the clinical situation presented. ${ }^{36}$

In conclusion, there is a need for more statistical data and, consequently, for carefully planned clinical tests to further evaluate effectiveness and safety of plasmapheresis treatment and of the combined techniques described in this narrative review.

\section{List of acronyms}

ARDS - acute respiratory distress syndrome COVID-19 - Coronavirus disease 2019

CRP - C-reactive protein

ICTV - International Committee on Taxonomy of Viruses

MODS - multiple organ dysfunction syndrome

SARS-CoV-2 - Severe acute respiratory syndrome coronavirus 2

\section{Authors contributions}

Ilmira R. Gilmutdinova, Yu M. Yakovlev, Petr S. Eremin and Anatoliy D. Fesun have contributed equally to this work.

\section{Acknowledgments}

The Authors thank the A\&C M-C Foundation for Translational Myology, Padova, Italy and the Federal State Budgetary Institution "National Medical Research Centre for Rehabilitation and Balneology" of the Ministry of Health of Russia, Moscow, Russia for supporting this publication.

\section{Funding None}

\section{Conflict of Interest}

The authors declare they have no financial, personal, or other conflicts of interest.

\section{Ethical Publication Statement}

We confirm that we have read the Journal's position on issues involved in ethical publication and affirm that this report is consistent with those guidelines.

\section{Corresponding Author}

Gilmutdinova Ilmira R., Federal State Budgetary Institution "National Medical Research Centre for Rehabilitation and Balneology" of the Ministry of Health of Russia, Moscow, Russia.

ORCID iD: 0000-0001-6743-2615

Email: gilm.ilmira@mail.ru

Emails and ORCID iD of Coauthors

Maksim Yu. Yakovlev: masdat@mail.ru

ORCID iD: 0000-0002-5260-8304

Petr S Eremin: ereminps@gmail.com

ORCID iD: 0000-0001-8832-8470

AnatoliyD Fesun: nmicrk@nmicrk.ru

ORCID iD: 0000-0003-3097-8889

\section{References}

1. Ma J, Xia P, Zhou Y, et al. Potential effect of blood purification therapy in reducing cytokine storm as a late complication of critically ill COVID-19. Clin Immunol 2020;214:108408. doi: 10.1016/j.clim.2020.108408.

2. Chen C, Zhang XR, Ju ZY, He WF. Advances in the research of cytokine storm mechanism induced by Corona Virus Disease 2019 and the corresponding immunotherapies. Zhonghua Shao Shang Za Zhi 2020;36(0):E005. doi: 10.3760/cma.j.cn501120-20 200224-00088

3. Guo YR, Cao QD, Hong ZS, et al. The origin, transmission and clinical therapies on coronavirus disease 2019 (COVID-19) outbreak - an update on the status. Mil Med Res 2020;7(1):11. doi: 10.1186/s40779-020-00240-0

4. Liu Y, Zhang C, Huang F, et al. 2019-novel coronavirus (2019-nCoV) infections trigger an exaggerated cytokine response aggravating lung injury. 2020. http://www.chinaxiv.org/abs/202 $\underline{002.00018}$ Accessed 18 Feb 2020

5. Huang C, Wang Y, Li X, et al. Clinical features of patients infected with 2019 novel coronavirus in Wuhan, China. Lancet. 2020;395(10223): 497-506. doi: 10.1016/S0140-6736(20)30183-5

6. Mehta P, McAuley DF, Brown M, et al. COVID19: consider cytokine storm syndrome and immunosuppresion. Lancet 2020;395(10229):1033-4.

7. Tabibi S, Tabibi T, Conic RRZ, et al. Therapeutic Plasma Exchange: A potential Management Strategy for Critically Ill COVID-19 Patients. J Intensive Care Med 2020;35:827-35. doi: 10.1177/0885066620940259

8. Yang X-H, Sun R-H, Zhao M-Y, et al. Expert recommendations on blood purification treatment protocol for patients with severe COVID-19. Chronic Dis Transl Med 2020 Apr 28;6(2):106-114. doi: 10.1016/j.cdtm.2020.04.002

9. Keith P, Day M, Perkins Let al. A novel treatment approach to the novel coronavirus: an argument for the use of therapeutic plasma exchange for fulminant COVID-19. Crit Care 2020;24:128. doi: 10.1186/s13054-020-2836-4

10. Avdeev SN. Acute respiratory distissue syndrome. Consilium medicum 2005;7: 330-8. (in russ.).

11. Pourahmad R, Moazzami B, Rezaei N. Efficacy of Plasmapheresis and Immunoglobulin Replacement Therapy (IVIG) on Patients with COVID-19. SN Compr Clin Med. 2020 Jul 31:1-5. doi: 10.1007/s42399-020-00438-2.

12. Kalinin NN. Clinical application of extracorporeal treatment methods. Publishing House: Trackpore Technology CJSC, 2006, pp. 168. (in russ.)

13. Ragimov AA, Poreshina SA, Salimov EL. Plasmapheresis in systemic inflammatory response at P14. Practical medicine 2008;124. (in russ.).

14. Madore F. Plasmapheresis. Technical aspects and indications. Crit Care Clin 2002;18:375-92. 
15. Hirano R, Namazuda K, Hirata N. Double filtration plasmapheresis: Review of current clinical applications. Ther Apher Dial 2020. doi: 10.1111/1744-9987.13548

16. Navarro-Martínez R, Cauli O. Therapeutic Plasmapheresis with Albumin Replacement in Alzheimer's Disease and Chronic Progressive Multiple Sclerosis: A Review. Pharmaceuticals (Basel). 2020;13:28. doi: 10.3390/ph13020028

17. Sergent SR, Ashurst JV. Plasmapheresis. 2020 Jul 15. In: StatPearls [Internet]. Treasure Island (FL): StatPearls Publishing; 2020 Jan.

18. Schwartz J, Padmanabhan A, Aqui N, et al Guidelines on the Use of Therapeutic Apheresis in Clinical Practice-Evidence-Based Approach from the Writing Committee of the American Society for Apheresis: The Seventh Special Issue. J Clin Apher 2016;31:149-62.

19. Clark WF, Huang SS. Introduction to therapeutic plasma exchange. Transfus Apher Sci 2019;58:2289. doi: 10.1016/j.transci.2019.04.004.

20. Sokolov AA, Popov AV. Cascade plasmofiltration: method characteristic and equipment selection. Tverskoi meditsinskii zhurnal 2017;5:46-58. (in russ.)

21. Sokolov AA, Tishko VV, Belskikh AN, Esipov AV. Modern extracorporal technologies: prospects of use. Gospital'naya medicina: nauka i praktika. 2019; 1: 32-41. (in russ.)

22. Rubtsov MS, Shukevich DL. Modern extracorporeal methods for critical conditions caused by systemic inflammatory response (review). Anesteziologiya i reanimatologiya. 2019;(4): 20-30. (in russ.) doi: 10.17116/ anaesthesiology201904120

23. Shen C, Wang Z, Zhao F, et al. Treatment of 5 Critically Ill Patients With COVID-19 With Convalescent Plasma. JAMA 2020 Mar 27. doi: 10.1001/jama.2020.4783.

24. Bloch EM, Shoham S, Casadevall A, et al. Deployment of convalescent plasma for the prevention and treatment of COVID-19. J Clin Invest 2020 Apr 7. pii: 138745. doi: 10.1172/JCI138745.

25. Chen L, Xiong J, Bao L, Shi Y. Convalescent plasma as a potential therapy for COVID-19. Lancet Infect Dis 2020;20:398-400. doi: 10.1016/S1473-3099(20)30141-9

26. Murphy M, Estcourt L, Grant-Casey J, Dzik S. International Survey of Trials of Convalescent Plasma to Treat COVID-19 Infection. Transfus Med Rev 2020:S0887-7963(20)30031-6. doi: 10.1016/j.tmrv.2020.06.003

27. Rajendran K, Narayanasamy K, Rangarajan J, et al. Convalescent plasma transfusion for the treatment of COVID-19: Systematic review. J Med Virol
2020 May 1. doi: 10.1002/jmv.25961

28. Seghatchian J, Lanza F. Convalescent Plasma, an Apheresis Research Project Targeting and Motivating the Fully Recovered COVID 19 Patients: A Rousing Message of Clinical Benefit To Both Donors and Recipients Alike. Transfus Apher Sci 2020 Apr 23 : 102794. doi: 10.1016 /j.transci.2020.102794

29. Cao W, Li T. COVID-19: towards understanding of pathogenesis. Cell Res 2020 Apr 28 : 1-3. doi: 10.1038/s41422-020-0327-4

30. Tang N, Li D, Wang X, Sun Z. Abnormal coagulation parameters are associated with poor prognosis in patients with novel coronavirus pneumonia. J Thromb Haemost 2020;18:844-847. doi: 10.1111 jth.14768

31. Sloan SR, Andrzejewski C, Aqui NA, et al. Role of therapeutic apheresis in infectious and inflammatory diseases: Current knowledge and unanswered questions. J Clinl Apher 2014;30:259 64. doi: 10.1002/jca.21370.

32. Yang $\mathrm{XH}$, Sun RH, Zhao MY, et al. Expert recommendations on blood purification treatment protocol for patients with severe COVID-19: Recommendation and consensus. Chronic Dis Transl Med 2020 Apr 28. doi: 10.1016/j.cdtm. 2020.04.002.

33. Keith P, Wells A, Hodges J, et al. The therapeutic efficacy of adjunct therapeutic plasma exchange for septic shock with multiple organ failure: a single center retrospective review. 2020. Preprint at https://www.researchsquare.com/article/rs16022/v1. doi: 10.1186/s13054-020-03241-6

34. Shi H, Zhou C, He P, et al. Successful treatment of plasma exchange followed by intravenous immunogloblin in a critically ill patient with 2019 novel coronavirus infection. Int $\mathbf{J}$ Antimicrob Agents 2020:105974. doi: 10.1016/j.ijantimicag. 2020.105974

35. https://www.fda.gov/news-events/pressannouncements/coronavirus-covid-19-update-fdaauthorizes-blood-purification-device-treat-covid$\underline{19}$.

36. Central Board of the Scientific Society of Experts of Extracorporeal Blood Treatment in Intensive Care. Interim recommendations on the use of methods of extracorporeal haemocorrection in the complex treatment of patients with new coronavirus disease COVID-19. http://www.rusnephrology.org/ wpcontent/uploads/2020/04/extracorp_hemocorrec t.pdf

Submission: June 5, 2020

Revision received: September 2, 2020 Accepted for pubblication: September 2, 2020 